\title{
Coronal dimming and the coronal mass ejection onset
}

\author{
R. A. Harrison ${ }^{1}$, P. Bryans ${ }^{1,2}$, G. M. Simnett ${ }^{3}$, and M. Lyons ${ }^{3}$ \\ 1 Space Science and Technology Dept., Rutherford Appleton Laboratory, Chilton, Didcot, Oxfordshire OX11 0QX, UK \\ 2 Department of Physics and Applied Physics, University of Strathclyde, Glasgow, Scotland, UK \\ 3 School of Physics and Space Research, Birmingham University, Birmingham B15 2TT, UK \\ Received 1 July 2002 / Accepted 21 January 2003
}

\begin{abstract}
A set of five observations of extreme-ultraviolet (EUV) coronal dimming associated with coronal mass ejection (CME) activity is examined. Using spectroscopic data, plasma characteristics across a broad range of temperatures from $20000 \mathrm{~K}$ to 2 million $\mathrm{K}$ are determined. The dimming events are found to coincide in time, and to coincide spatially, with the projected onset times and locations of the associated CMEs. The spectral data confirm that the dimming is due to massloss, and not temperature variations. The actual mass-loss calculated from the degree of dimming, using two different methods, shows that the extracted mass in each case, is of the same order as the mass of the associated CME. In some cases, the EUV observations are limited to relatively small regions under the CME events and it is expected that we do not witness the massloss associated with the entire event, for these. However, we believe that this analysis has provided a method for locating the source region of the trigger for a CME eruption, and that the dimming characteristics can be used to distinguish between onset processes of the CME. In particular, the gradual nature of the dimming process, which takes place over several hours, suggests that either the CME has a continuous driver rather than a sudden impulsive onset, or the low coronal response to a CME extends over a long period.
\end{abstract}

Key words. Sun: activity - Sun: atmosphere - Sun: UV radiation - Sun: coronal mass ejection (CMEs)

\section{Why EUV spectroscopic observations of CME onsets?}

We have known about the solar Coronal Mass Ejection (CME) phenomenon for three decades and have long observational data-sets of these events from coronagraphs aboard Skylab (1972-3), the Solar Maximum Mission (1980-89), P78-1 (1979-1985) and the Solar and Heliospheric Observatory (SOHO; 1995 to date) (see reviews by Hundausen 1997 and Howard et al. 1997). These observations have provided remarkable insight into the nature of CME events and have given us some of the most spectacular images in modern day astronomy. We have recognised the importance of CMEs to understanding solar/stellar mass-loss processes, to the understanding of coronal structure and evolution, and the generation of geomagnetic events. We have long been able to construct mathematical models, which mimic the eruption of magnetic systems in a model corona (e.g. Low 1981; Wolfson 1982; Priest 1988). However, even with the availability of direct coronal imaging (in X-rays and Extreme Ultraviolet [EUV]) combined with the coronagraph observations of CMEs, and armed with models of eruptive processes, we do not fully understand the CME onset mechanisms.

Part of the problem is the fact that we do not have a method for coronal magnetography. We must look for the source of

Send offprint requests to: $\mathrm{R}$. A. Harrison,

e-mail: r.harrison@rl.ac.uk
CMEs using direct observation of the coronal plasmas using $\mathrm{X}$-ray and EUV techniques, and infer something about the nature of the magnetic fields of the corona through the examination of the plasma topology and evolution.

Direct coronal images combined with coronagraph observations of CMEs have, indeed, demonstrated that it is often extremely difficult to identify any eruptive feature associated with the CME process. The drama observed in the outer corona is often not reflected in a dramatic event in the low corona. However, when prominence eruptions are associated with CMEs, we can clearly identify the ascending prominence plasma. When there is an associated flare, we can identify it in a range of wavelengths. However, we find it extremely difficult to identify the source of the ejected coronal mass itself, in the low corona. In addition, many CMEs are not associated with flare activity or prominence eruptions.

In short, the combination of direct coronal imaging and coronagraph observations has not provided the data which, many anticipated, would solve the CME onset problem. Thus, our choice of models of CME onsets remains remarkably open and we are not in a position to describe accurately even the basic magnetic structure of the CME source region.

Thus, we are faced with an observational problem. We must find an observational tool for detecting the source structure of a CME. For this reason, we resort to EUV spectroscopy.

In the first instance, monitoring a large region of the low corona, with a selection of emission lines, provides a method 
for detecting the CME source region and onset over a wide range of temperatures simultaneously.

In addition, the use of EUV spectroscopy provides plasma diagnostic parameters such as temperature, density and plasma velocity. Such parameters can be used to identify a variety of features. We can clearly identify the loss of mass from a region of the low corona through the use of emission line comparisons. We can identify the generation of jets or heating at the sites of magnetic reconnection, through the analysis of line shapes and the effects at a wide range of temperatures. We can determine the relationship between coronal plasma at a wide range of different temperatures. Much of this can only be determined unambiguously through the use of spectroscopy and such information is essential to determine the processes leading to the eruption.

\section{EUV dimming}

Since 1996/7, dimming of the low corona, as observed in the EUV and X-rays, has been reported in association with many CMEs, using observations from the Soft X-ray telescope (SXT) on the Japanese Yohkoh spacecraft, the EUV Imaging Telescope (EIT) on SOHO, and the Coronal Diagnostic Spectrometer (CDS) on SOHO. For a review of the dimming phenomenon, see Sect. 1 of Harrison \& Lyons (2000) and for individual studies see Sterling \& Hudson (1997), Zarro et al. (1999), Harrison (1997) \& Harrison \& Lyons (2000 - henceforth Paper I), and references therein. However, despite the detection of dimming under many CME events, the relationship between the dimming and the CME activity remains unclear. Broad-band imaging (e.g. SXT/Yohkoh and EIT/SOHO) cannot distinguish unambiguously between temperature and density changes as the physical process characterised by the observed dimming. However, this has been done using spectral observations (Harrison \& Lyons 2000), which showed that the dimming is due to mass loss from the emitting region. However, there are relatively few dimming events where spectral data have been available, and it is the purpose of this paper to extend this effort considerably.

However, we do wish to stress that these are dimming events, as distinct from the events known as EIT waves or coronal Moreton waves (see e.g. Thompson et al. 1999; Biesecker et al. 2002). There is some confusion about the relationship between dimming events and the EIT waves, so we raise this issue here. The EIT or coronal Moreton waves are identified as rapidly expanding disturbances in the corona, often, but not always, associated with CME onsets. They are usually identified using image subtraction techniques, which means that the propagation is identified through the enhancement and depletion of intensity between full-Sun EUV images. On the other hand, the coronal dimming events are also identified by EUV (and X-ray) image subtraction. However, they are well-defined dimming regions located under CMEs, whereas the EIT waves are rapidly expanding disturbances crossing the solar surface. Some CME events display coronal waves and EUV dimming, but they are distinctly different phenomena.

One might take the view that since a CME involves the expulsion of a large amount of matter from the corona that it is logical that an inspection of the underlying low corona will display dimming, i.e. mass is lost, so we expect dimming. Indeed, this is so, but one has to consider what the dimming phenomena in itself can tell us. As mentioned above, the effects in the low corona of a CME can be extremely subtle; it is not easy to see signatures of the CME onset in the low corona. However, dimming is one such feature, and, thus, the authors regard the investigation of the dimming process as a major step in the investigation of the physics of CME onsets.

Of the three instruments listed above, the SXT and EIT instruments are broad-band imagers. For the reasons given above, we require plasma diagnostic information, and believe that it is better to use a spectrometer operating in the EUV or X-ray region. Thus, for the current work, we use the CDS instrument to identify and characterise the plasma characteristics of the dimming events.

The basic EUV observation scheme, which is described in the next section, is labelled Joint Observing Programme (JOP) 67. The observations are supported by the coronagraph also on board SOHO.

The basic desire, then, is to obtain spectral observations, and therefore, plasma diagnostic information, on the source regions of CMEs at the time of onset. The JOP 67 scheme has been run on many occasions and, to date, a number of periods have been identified where we have CME observations with supporting CDS observations of what we believe to be the underlying CME source region. One of the events has been presented previously (Paper I).

In this paper, we present and summarise the data from five CME/dimming events. We identify the coronal dimming and present an analysis of the dimming process. This is used to determine the nature and evolution of plasma characteristics in the dimming region.

\section{The observation scheme}

The object of the observing sequence used for this work is to monitor a large volume of the limb corona, that is, a large latitudinal span including areas on the disc and off-limb plasma well out of the plane of the sky, using a range of emission lines representing a large range of temperatures.

The CDS instrument is a double spectrometer operating in the 150-800 $\AA$ EUV range. It allows the detection and analysis of spectral emission lines from numerous trace ions in the solar atmosphere, with spatial, temporal and spectral resolving elements of down to 2 arcsec, a few seconds and $0.08 \AA$, respectively (see Harrison et al. 1995 for a full description). The selected wavelength region contains emission lines which come from ions with characteristic temperatures in the range $20000 \mathrm{~K}$ to over 2 million $\mathrm{K}$, thus making this particular wavelength region extremely valuable for studies of the solar atmosphere where large temperature variations exist.

The details of the observation scheme used in this work are described in detail in Paper I. The basic observation is a $4 \times 12$ arcmin field of view made up of three $4 \times 4$ arcmin rastered images taken in six emission lines. The six lines used, and their temperatures of formation, are given in Table 1. For each image pixel, we return data for 15 spectral bins across 
Table 1. Emission lines used for this study.

\begin{tabular}{lll}
\hline \hline Ion & Wavelength Range $(\AA)$ & Temperature $(\mathrm{K})$ \\
\hline He I 584.33 & $583.5-585.2$ & 20000 \\
O V 629.73 & $629.0-630.6$ & 250000 \\
Mg IX 368.06 & $367.6-368.6$ & 1000000 \\
Si X 347.40 & $347.1-348.1$ & 1300000 \\
Si X 356.04 & $355.6-356.6$ & 1300000 \\
Fe XVI 360.76 & $360.3-361.3$ & 2000000 \\
\hline
\end{tabular}

each of the six emission lines; the resulting ranges are shown in the table. The number of returned lines and the numbers of pixels across each line were kept to a minimum, and only bright lines were selected, to enable as rapid a cadence as possible. Nevertheless, the sequence cadence is $50 \mathrm{~min}$. Two of the selected lines, the Si X pair, provide a measure of density for million $\mathrm{K}$ plasmas when their intensity ratios are considered (see Mason et al. 1997).

A number of extended observing runs have been made with the JOP 67 sequence. A search through the runs has revealed a number of periods where the CDS field is apparently looking at the source region of a CME as detected in the outer corona above, using the Large Angle Spectroscopic Coronagraph (LASCO; see Brueckner et al. 1995) also aboard SOHO. Other instruments, both space and ground-based were involved in the JOP 67 runs, but the current analysis concentrates on the identification of CMEs and the analysis of the associated dimming and, thus, involves only the LASCO and CDS data.

\section{The events}

We treat the events in chronological order starting with the event described in Paper I. This event is used to demonstrate the techniques employed for all events. In this event, we detect significant, long-duration dimming from coronal emission lines from a region off the western limb.

\subsection{July 16, 1997}

Figure 2 of Paper I shows the CME event of July 16, 1997. The CDS observations started at 10:18 UT and ran for some 12 hours during which time the CME was detected by LASCO. Figure 1 (of the current paper) shows the dimming as detected in the 1 million $\mathrm{K} \mathrm{Mg}$ IX emission line. The raw images show a weak active region crossing the equator but little else. Differencing the images, by subtracting the first frame from each subsequent image (lower panel of Fig. 1) shows the dimming clearly. The extent and position angle of the dimming event are identical to those of the overlying CME (shown in Paper I).

A white box denotes the area where we perform a detailed analysis. It encloses the dimming area and includes some $31 \times 88$ pixels of size $2 \operatorname{arcsec} \times 1.68$ arcsec. For these pixels, we sum the intensities from each emission line and study the timeintensity profiles for the region. After summing, a background level is estimated by making Gaussian fits to the data, and the
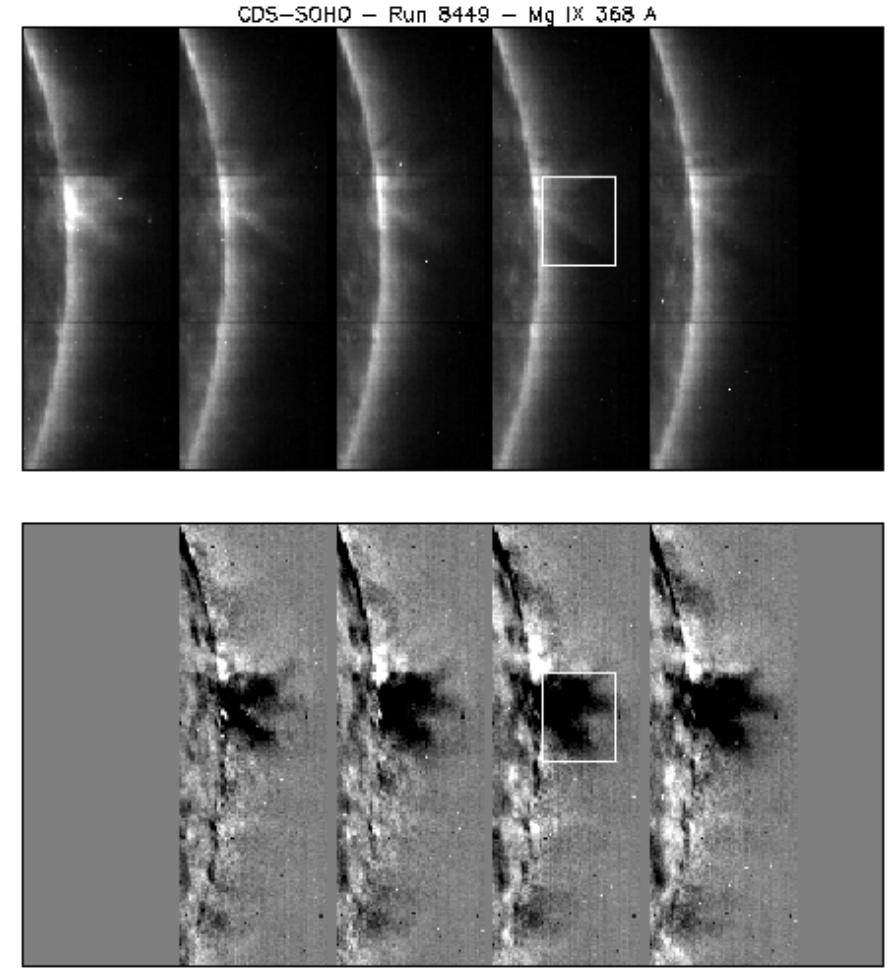

Fig. 1. The dimming event of July 16, 1997 detected in the 1 million K Mg IX emission line at $368 \AA$. The top panel shows the images, at 50 min cadence. The bottom panel shows the same data with the first frame subtracted from all images. The dimming can be seen clearly. The white box indicates the pixel area used for detailed analysis.

background is removed. Thus, we can explore the characteristics of the individual emission lines. Figure 2 shows the timeintensity profiles of the six lines for the box of Fig. 1. Error bars are included in the plots, though they are mostly smaller than the plotted symbol.

These figures are similar to Figs. 8 to 10 of Paper I. However, the selected pixel area is larger in this work, to cover the full dimming region, and background was not removed from the Paper I analysis (under the assumption that the emission line dominated the intensity). This means that the error bars of Fig. 2 are much smaller than the previous paper, and we have highlighted only the effects from the emission lines.

Before considering the time-intensity profiles, we must define what we accept as a dimming event. The following rules have been applied:

- A dimming event must include at least two consecutive rasters with a decline in intensity which is greater than three times the calculated error, or show a clear drop in average intensity (by more than three times the calculated error) for a sustained period;

- It is preferable to detect a minimum, though not essential (the decline may extend beyond the observations), and this can be determined by the occurrence of at least two consecutive intensities which show either a constant value or a rise.

The Mg IX profile shows a gradual decline from the start (10:18 UT), falling by about 30\% over almost 6 hours, and by $41 \%$ by the end of the observation. Although the curve levels off a little, the overall trend is downward even to the end of 

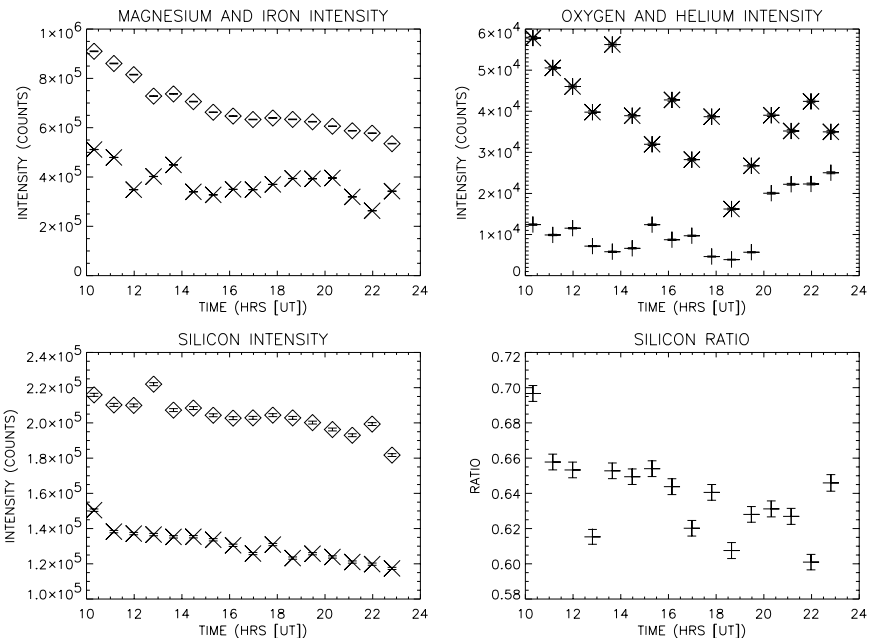

Fig. 2. The time-intensity profiles of the six emission lines for the dimming area (white box) of the July 16, 1997 event. The top left panel shows the Mg IX (diamonds) and Fe XVI (crosses) data (the Fe XVI intensities are multiplied by a factor 10), the top right panel shows the O V (stars) and He I (crosses) data. The bottom left panel shows the Si $347 \AA$ (diamonds) and $356 \AA$ (crosses) data; the ratio of these two is given in the lower right panel.

the observation. The Fe XVI data show a similar decline. Note that the Fe XVI intensity in Fig. 2 is multiplied by a factor of 10 for ease of display. We note that this decline was not detected by Paper I, which may be due to (i) the larger pixel area used here, (ii) changes in the background intensities (which were not removed in Paper I), or (iii) the relatively low intensity of the Fe XVI data.

The He I and O V data show great variations. The widely varying intensities show no coherent dimming event at these temperatures. Thus, no dimming events are included for the $\mathrm{He} \mathrm{I}$ and $\mathrm{O} \mathrm{V}$ in the analysis. However, the Si X profiles show a similar story to the Mg IX intensity, though the decline is to a lesser degree.

The Si X energy level populations are influenced by density, over a specific density range, because of the presence of a metastable level and this forms the basis of the work due to Mason et al. (1997) from which we can estimate a density from the Si X 356/347 intensity ratio. The ion has a characteristic temperature of 1.3 million $\mathrm{K}$, so the density is, in effect, for plasmas at these temperatures. The lower-right panel of Fig. 2 shows a peak ratio of 0.7 at the start of the observation, relating to a $\log$ density of $8.6 \mathrm{~cm}^{-3}$. The general trend is a clear fall, with an associated fall in $\log$ density to a value nearer to $8.4 \mathrm{~cm}^{-3}$.

We note that all coronal lines $(\mathrm{Mg}, \mathrm{Si}, \mathrm{Fe})$ show a decrease in intensity with time across the observation. This is consistent with a density or filling factor decrease rather than a temperature change. If the Mg IX intensity fall was due to heated plasma emitting less and less at the million $\mathrm{K}$ band, then the $\mathrm{Si} \mathrm{X}$ and then the Fe XVI intensities would be expected to rise subsequently. Also, if 2 million $\mathrm{K}$ plasma was cooling, one might expect the million $\mathrm{K}$ bands to show an increase with time. All bands from 1 million to 2 million $\mathrm{K}$ show the same decline.
In Table 2, we summarise the characteristics of these profiles and the general properties of the event. Initially, for each line we identify the degree of dimming, defined as the percentage change in intensity from the apparent onset to the leveling off of the dimming. In this case, that is the start and end of the data-set for most lines. Column two, shows the percentage decline in intensity and the third column shows the onset time of the dimming and the time of the minimum intensity.

Of course, the precise onset of dimming, and the timing of the minimum, are somewhat subjective, but the aim is to identify the general trends and properties. In addition, we note that for some events the dimming is either underway from the first raster or is still evident at the last raster. For such events, we can only put limits on the dimming timing and indicate this in the table.

Table 2 includes an estimate of the density change from the Si X ratio, which in this case showed a clear downward trend but with some variation. We also calculate a mass loss, which would be consistent with the dimming event. This is calculated in two ways, which we denote the DEM method and the Si X ratio method.

Using the change in density estimated from the Si X ratio, say $n_{e 1}$ to $n_{e 2}$, we can calculate a mass by taking the boxed area, which is $N_{x} \times N_{y}$ pixels, and assuming that the source region is as deep as it is wide. Given the volume and density change, the mass can be readily estimated, i.e.

$\left(n_{e 1}-n_{e 2}\right)\left(N_{x} L_{x} N_{y} L_{y} N_{x} L_{x}\right) m_{\mathrm{p}}$

where $m_{\mathrm{p}}$ is the mass of the proton, and $L$ is the conversion from pixel size to distance on the solar surface.

The drawbacks of this method are (i) that the Si X data can only detect changes in density for plasmas of a temperature near 1.3 million $\mathrm{K}$ and thus cannot see the cooler and hotter emissions, and (ii) we have assumed something about the depth of the dimming region, with little justification. However, the calculated mass should be considered to be a reasonable figure for a basic comparison to LASCO masses, for example.

For the other method, we try to take the emissions at all temperatures into account, by making use of a Differential Emission Measure (DEM). We use a "standard" DEM used by Raymond \& Doyle (1981), as displayed in their Fig. 3. In effect, we are accepting a standard distribution of the amount of matter at the different temperatures, i.e. a contribution function for the emission lines we are using. For example, for the He I, O V, Mg IX and Fe XVI lines at $20000 \mathrm{~K}, 250000 \mathrm{~K}, 1$ million K and 2 million $\mathrm{K}$, the relative contributions are given as 47,1 , 18 and 32, in arbitrary units, based on the Raymond \& Doyle quiet Sun DEM.

If we accept that the intensity is proportional to the square of the density for each line, since the excitation is dependent on collisional processes, then the drop in intensity for each line indicates a change in density at each temperature. Thus, the relative contributions, given above, fall accordingly. If we accept a starting density, such as that given by the Si X ratio, and assume that the contribution from each line is in proportion to the Raymond and Doyle DEM, then the relative change in intensity for the line set can be used to derive the mass lost. We have to make the same assumption for the emitting volume as for the 
Table 2. The Event of July 16, 1997 (CDS Run 8449).

\begin{tabular}{|c|c|c|}
\hline Line & Dimming & Onset/Minimum \\
\hline He I $584 \AA$ & $-0 \%$ & $-1-$ \\
\hline No clear event. & & \\
\hline O V $629 \AA$ & $-0 \%$ & $-/-$ \\
\hline Similar to He I. & & \\
\hline Mg IX $368 \AA$ & $-41 \%$ & $\leq 10: 18 / \geq 22: 48 \mathrm{UT}$ \\
\hline Continuous decline. & & \\
\hline Si X $347 \AA$ & $-16 \%$ & $\leq 10: 18 / \geq 22: 48 \mathrm{UT}$ \\
\hline Si X $356 \AA$ & $-22 \%$ & $\leq 10: 18 / \geq 22: 48$ UT \\
\hline Fe XVI $360 \AA$ & $-49 \%$ & $\leq 10: 18 / 21: 58 \mathrm{UT}$ \\
\hline
\end{tabular}

DENSITY: 11\% fall in Si X ratio, 10:18 to 22:48 UT. Ratio fluctuates but downward trend from 0.70 to 0.62 . Consistent with $37 \%$ density drop, $\log _{10}=8.6$ to $8.4\left(\mathrm{~cm}^{-3}\right)$.

MASS LOSS: DEM method $=4.3 \times 10^{10} \mathrm{~kg}$, Si X ratio method $=$ $1.3 \times 10^{11} \mathrm{~kg}$.

\section{POSITION ANGLE: 256-284.}

LASCO EVENT: Small, bright ejection detected from 13:30 UT. Projected onset 10:50-11:20 UT. Position angle 259-275 . Mass $5 \times 10^{10} \mathrm{~kg}$.

COMMENT: Good CDS and LASCO coverage. Events appear to be well related.

Si X method. Of course, one drawback of this method is the fact that we have had to make an assumption about the initial DEM which may not be correct.

The CME masses tabulated are calculated using LASCO white-light data. For each event discussed in this paper, the CME mass is calculated by comparing a LASCO C2 image at the peak of the event (i.e. when the CME is well within the C2 field) with a post-event image. Analysis of the brightness change between the two images assuming Thomson scattering reveals a total mass change. The calculations assume that the CME is in the plane of the sky; if the CME was out of the plane of the sky, the mass estimate would be low. However, for the purposes of this analysis, and in view of the fact that associated source regions appear to be near or on the limb, this is a fair assumption.

The dimming mass estimates, calculated from the Si X ratio and the DEM method, are perfectly valid, but do contain uncertainties. However, it is clear that the CME masses calculated from LASCO data are equally open to errors due to the assumption of ejection in the plane of the sky. Thus, the three mass estimates are reasonable for comparison but should be considered carefully. For the July 16, 1997 masses calculated from the dimming data, the DEM and Si X methods produce similar values which are within a factor of 3 of one another, and the CME mass from LASCO falls between the two values. Thus, we have good agreement and the suggestion is that the masses could be one and the same, i.e. the mass in the CME is the mass lost from the low corona indicated by the dimming.

Also in the table, we show the position angle of the dimming area. This also compares favourably with the location and spread of the associated CME, apparently confirming the intimate association between the dimming mass and the CME mass.

The projected CME onset time can be compared to the dimming profiles. In this case, the dimming started at or before 10:18 UT and the projected CME onset was in the window 10:50 to 11:20 UT. This suggests that the dimming started at least 32 minutes before the onset of the CME. However, if we consider the early acceleration of the CME, the possibility of the CME source being at a certain altitude prior to launch and the possibility of the source being slightly out of the plane of the sky, the uncertainty on the CME onset time is large enough to mean that we can claim a superb association, and, to all intents and purposes the data suggest that the CME and dimming onsets were coincident in time.

Another issue is the duration of the decline in EUV intensities. The profiles of Fig. 2 show a decline over many hours. However, LASCO measurements at 2.3-2.4 solar radii, above the dimming region, show that by $15: 32 \mathrm{UT}$, the outer coronal intensities are back to pre-event levels. This is after the most substantial decline in the Mg IX and Fe XVI emission lines, but the Mg IX emission line in particular shows further dimming. The Si X emission lines also show significant dimming after the LASCO observations return to "normal". This is a feature which we return to later.

We note that Paper I showed that there was no prominence eruption or flare associated with this event. Certainly, the ascent of a prominence would be seen as an increase and then decrease in the cooler He I and $\mathrm{O}$ V emission through the event. This is not seen.

We conclude from this particular event that the observations are consistent with the EUV dimming representing the source region, or part of the source region, of the subsequent CME. This claim is made on the basis of the coincident timing and location, as well as the mass and event sizes.

\subsection{May $8 / 9,1999$}

The May 8/9 event consists of the dimming of million K plasma off the east limb in association with the EUV observation of a prominence eruption and a LASCO CME. Although the EUV observations were somewhat truncated (only 5 mosaic images were made), the drop in intensity is clear.

The May 8/9, 1999 observations are shown, for the Mg IX $368 \AA$ and He I $584 \AA$ emission lines in Fig. 3, in the same format as Fig. 1. In the He I data one can see immediately the eruption of cool prominence material in the form of an ascending double loop structure. This is seen from the fourth image (at 24:00 UT), with the loops centred on position angle $73^{\circ}$. The Mg IX data show little evidence for the eruption in the raw intensity images but the differenced images reveal significant dimming of the hot plasma in the onset region, on and above the limb, at the same time.

Again, we show a boxed region in Fig. 3, which is used to study the emission line time-intensity profiles, which are shown in Fig. 4. On this occasion, the boxed area covers $120 \times 120$ pixels (each of size $2 \operatorname{arcsec} \times 1.68 \operatorname{arcsec}$ ), and we sum these in the same way as for the July 16 event. However, 
there were only five of the mosaic images produced for this observation. Still, the differenced Mg IX images of Fig. 3 show a significant area of dimming and the profiles of Fig. 4 show a continuous decline in many of the emission lines, with a drop after 23:00 UT. The characteristics are summarised in Table 3.

Using our definition of a dimming event, we find a significant decay in the Mg IX and both Si X lines from 23:09 UT and this was preceded by a fall in the He I intensity from the onset of the observation. However, no obvious dimming can be seen, which satisfies our definition of dimming, in the Fe XVI and $\mathrm{O} \mathrm{V}$ data. There are no increases in intensity to support the suggestion that the dimming of some lines is due to heating of plasma. Thus, as with the July 16 data, we conclude that mass is lost from the low corona.

We note that there is a significant peak in the $\mathrm{He} \mathrm{I}$ and $\mathrm{O} \mathrm{V}$ emission at 24:00 UT. This is undoubtedly due to the passage of the ascending prominence seen in the fourth frame of the upper panels of Fig. 3.

The projected CME onset was 23:31 UT across position angles $58^{\circ}$ to $78^{\circ}$. The dimming was first detected at 23:09 UT occupying position angles $65^{\circ}$ to $80^{\circ}$. The dimming and CME onset are clearly associated and, as with the July 1997 event, the error in the CME onset projection is such that the onsets are apparently identical in time. The EUV mass estimates are factors of 3.7 and 14 greater than the estimated CME mass. Again, given the errors associated with both the coronagraph and spectrometer mass calculations, an estimate within an order of magnitude can be considered to be very close.

As with the July 16 event, based on the coincident locations and event sizes, as well as the coincident timing and mass estimates, we conclude that the EUV dimmimg is highlighting the mass-loss from the CME source location.

\subsection{July 25, 1999}

The July 251999 observation shows a very significant depletion in the corona above a flare on the north-west limb under a large CME event. In this case the dimming was greatest in emission lines of temperatures $1.3 \times 10^{6} \mathrm{~K}$ and above.

The July 25, 1999 EUV observations are shown, for the He I $584 \AA$ and Fe XVI $360 \AA$ emission lines in Fig. 5. The observations were not made in this case using the three $4 \times$ 4 arcmin rasters, but consisted of a sequence of only one of the $4 \times 4$ arcmin rasters, taken every $16 \mathrm{~min}$. Despite the restricted area, we detected a mass ejection onset and a related flare. The Fig. 5 sequence shows $164 \times 4$ arcmin images in succession from top left to bottom right.

The flare can be seen quite clearly in the later images of the sequence, though the colour tables have been enhanced to bring out the response of the less intense features. The north-west limb is clearly visible, crossing each image from the middleleft to the bottom of the frame. The flare-arcade is visible on the limb in the He I and Fe XVI images The first brightenings are see in the 9th image which was taken at 13:12 UT and the flare is certainly underway by the next frame at 13:28 UT. However, above the flare-site, in the Fe XVI data, one can identify weakly emitting coronal loops. By 13:12 UT, these have
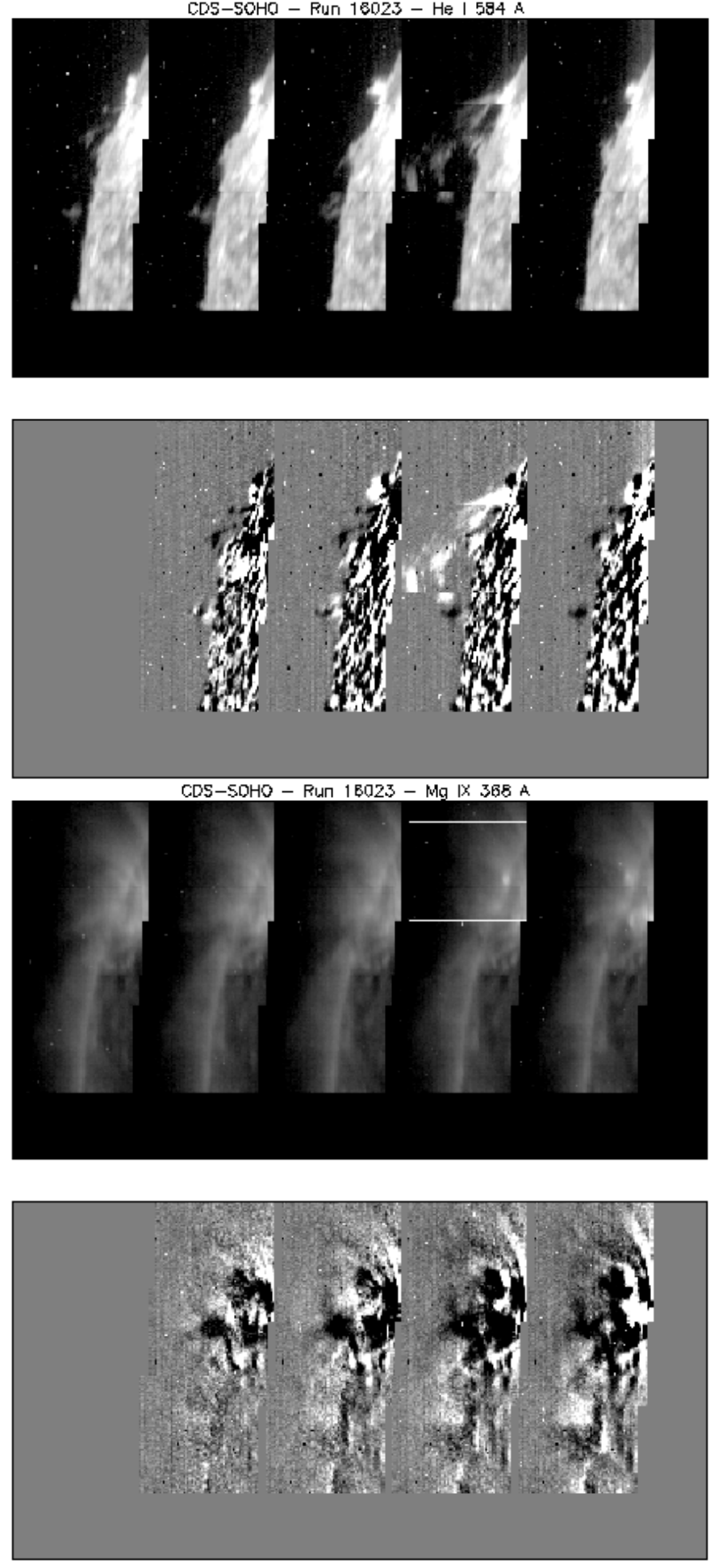

Fig. 3. The eruption and dimming events of May 8/9, 1999 detected in the $20000 \mathrm{~K} \mathrm{He}$ I $584 \AA$ and the 1 million K Mg IX $368 \AA$ emission lines, displayed in the same format as Fig. 1.

gone; the overlying corona appears to be void. This is a striking effect when one compares the off-limb corona of the first eight and last eight frames of the Fe XVI images of Fig. 5.

A white box marked in Fig. 5 shows the area of the corona where we investigate this depletion. The box is selected to be above the expanding loop system, at least for the majority of 

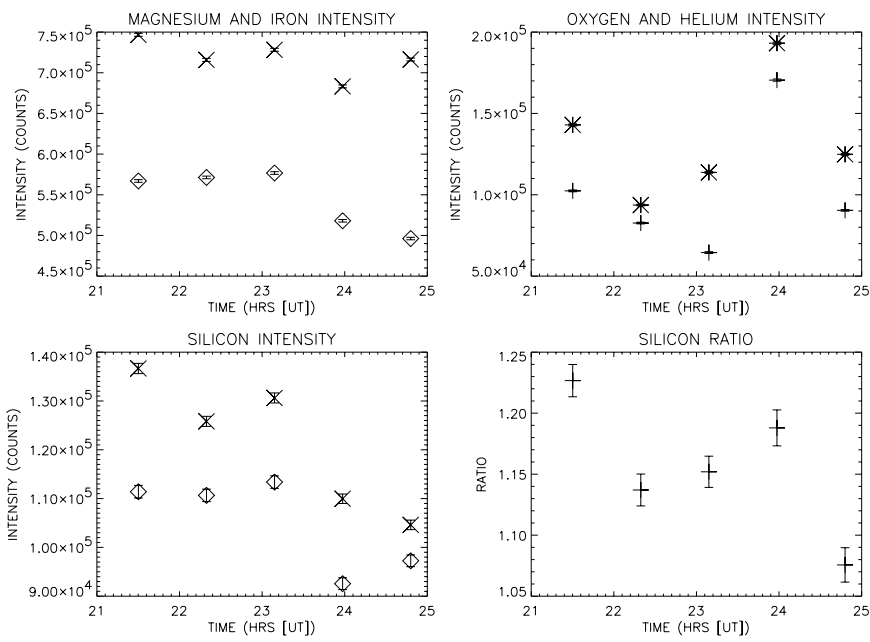

Fig. 4. The time-intensity profiles of the six emission lines for the dimming area of the May 8/9, 1999 event, displayed in a format similar to Fig. 2.

Table 3. The Event of May 8/9, 1999 (CDS Run 16023).

\begin{tabular}{lll}
\hline \hline Line & Dimming & Onset/Minimum \\
\hline He I 584 & $-37 \%$ & $\leq 21: 30 / 23: 09$ UT \\
Ascending loops. & & \\
O V 629 & $-0 \%$ & $-/-$ \\
Mg IX 368 & $-14 \%$ & $23: 09 / \geq 00: 47$ UT \\
Si X 347 & $-17 \%$ & $23: 09 / 23: 59$ UT \\
Si X 356 & $-24 \%$ & $23: 09 / \geq 00: 47$ UT \\
Fe XVI 360 & $0 \%$ & $-/-$ \\
\hline
\end{tabular}

DENSITY: Overall fall in Si X ratio about 1.24 to 1.08 , representing $37 \%$ density fall from $\log _{10}=9.3$ to $9.1\left(\mathrm{~cm}^{-3}\right)$. However, fall is not smooth.

MASS LOSS: DEM method $=1.1 \times 10^{12} \mathrm{~kg}$, Si $\mathrm{X}$ ratio method $=$ $4.2 \times 10^{12} \mathrm{~kg}$.

\section{POSITION ANGLE: $65-80^{\circ}$.}

LASCO EVENT: Relatively narrow CME. First detected at 00:26 UT. Projected onset 23:31 UT. Position angle 58-78 . Mass $3 \times 10^{11} \mathrm{~kg}$.

COMMENT: Good CDS and LASCO coverage. Events appear to be well related.

the event, to allow an analysis of the off-limb corona without influence from the brightening flare plasma. The box itself is $54 \times 75$ pixels in size (each pixel 2 arcsec by 1.68 arcsec).

The time-intensity profiles of the emission lines in this area are shown in Fig. 6. The Fe XVI data in particular, show a deep dimming over a period of about 1.5 hours, starting at about 12:39 UT, which is equivalent to the 8 th and 9 th frames of Fig. 5. Some dimming can be seen at the same time in the $\mathrm{Si} \mathrm{X}$ and Mg IX data, but the Fe XVI intensity drops out by as much as $89 \%$. However, late in the period, at about 15:00 UT, the corona brightens considerably, in most lines. This is due possibly to the uplifted post-flare loop system. As with the previous events, the nature of this event is summarised in Table 4.

The LASCO CME centre-line was at $296^{\circ}$, i.e. only $9^{\circ}$ from the centre of the CDS dimming. Thus, the positional association is very strong between the dimming and CME onset. In addition, the timing of the dimming, at 12:55 UT is within $25 \mathrm{~min}$ of the projected CME onset. The dimming and CME onset are clearly associated. However, the CME was a large event and most likely involved the eruption of multiple structures. When comparing this to the smaller $4 \times 4$ arcmin field of the CDS observation in this case, we must take care.

The best that we can conclude is the following. The EUV data show a significant dimming in the coronal data, coincident with a CME onset and location. At least some of the CME activity appears to originate from the region of the CDS observation. The timing and location are good evidence for a strong association. The EUV mass loss calculation may be significantly in error because it can only be done for a small area; the depleted region appears to be much larger and may extend well beyond the CDS field of view. However, the EUV dimming could be due to a mass loss equivalent to the mass detected in the CME. However, we acknowledge that this mass may only be part of the mass of the full eruption due to the complex nature of this event. Despite the complexity of this observation, the same basic association between dimming and CME activity is evident.

\subsection{February 19, 2000}

The February 19, 2000, event occurs under a loop-like CME on the western limb. A prominence eruption is detected and coronal dimming is identified from a compact region under the CME, especially in the 2 million K Fe XVI line.

The EUV observations are shown, for the He I $584 \AA$ (raw) and Fe XVI $360 \AA$ (differenced) emission lines in Fig. 7. For this sequence, only two of the $4 \times 4$ arcmin rasters were used to cover a region $4 \times 8$ arcmin square, with a cadence of $32 \mathrm{~min}$. In Fig. 7 , we show only one of the $4 \times 4$ arcmin fields. The He I data reveal an extremely active set of cool structures with some jet-like eruptive activity, similar to that reported by Harrison et al. (2001). The observation started at 07:02 UT. Apart from the clear motion of the He I emitting strutures, we also witness an extreme brightening in the second frame of the He I images, at 07:51 UT.

The differenced Fe XVI data show an extraordinary, compact dimming feature from the same region. In most of the dimming illustrations in this paper, we use differencing from a pre-event image. Here, we use a running difference because the first frame is so bright in the Fe XVI data. The initial intensity drop is dramatic and it is followed by continuing depletion as indicated by the subsequent frames. The depletion region is quite well defined and compact, especially between the first two frames. The white box of Fig. 7, which covers an area of $60 \times 100$ pixels of size $2 \operatorname{arcsec} \times 1.68$ arcsec, shows the area in which we examine the line intensities. The intensity profiles are shown in Fig. 8.

As the Fig. 8 time-intensity profiles indicate, the intensities of all six lines show significant dimming, i.e. across the entire temperature range. The Fe XVI intensity curve of Fig. 8 shows a bright initial intensity, with a large drop in intensity, followed by a gradual decay to the end of the observation. The Mg IX 


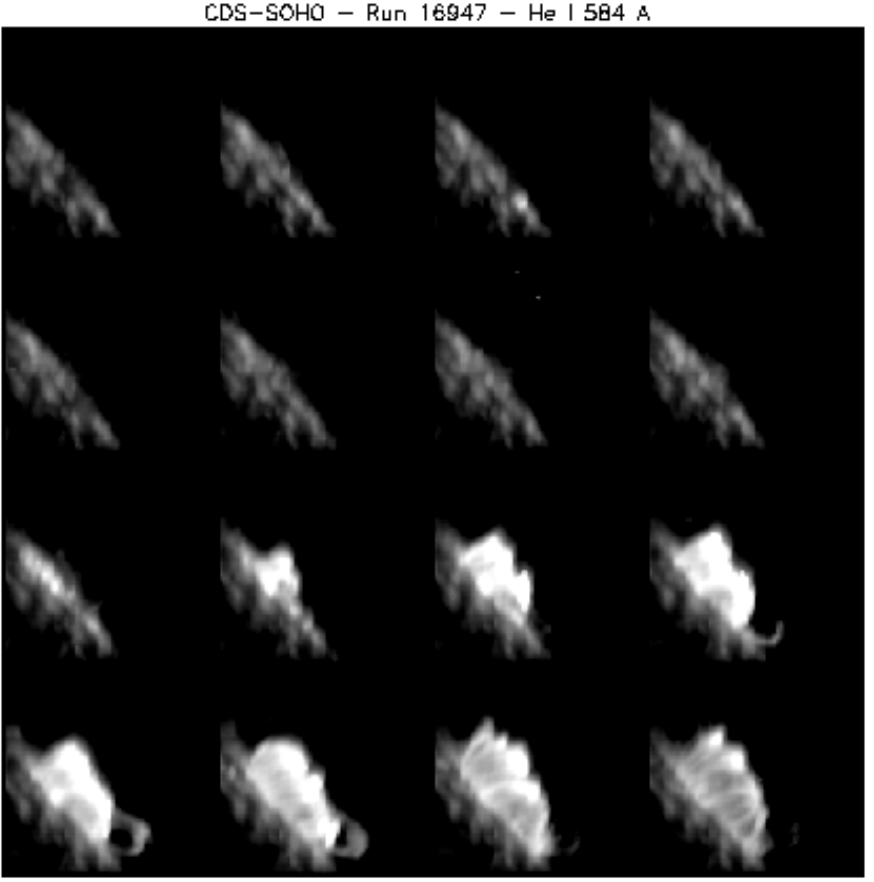

CDS-SOHO - Run 16947 - Fe XVI 360 A

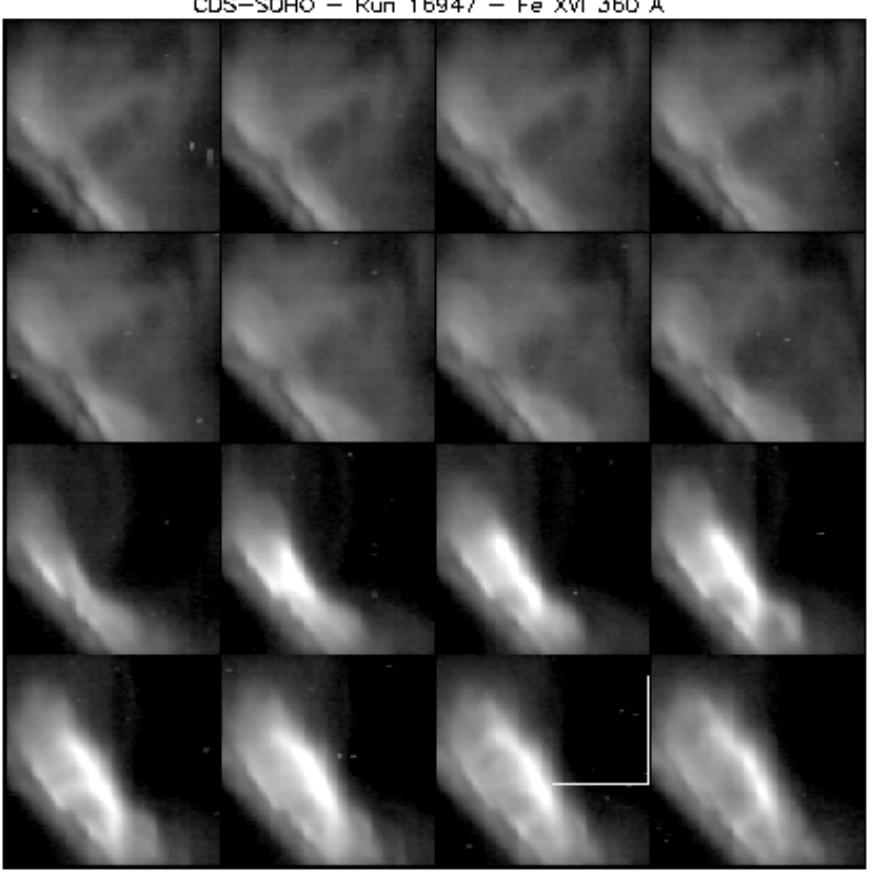

Fig. 5. The event of July 25, 1999 detected in the 20000 K He I emission line at $584 \AA$ (upper panel) and the 2 million K Fe XVI emission line at $360 \AA$ (lower panel). Unlike the other datasets, this event was detected using only a $4 \times 4$ arcmin field. The sequence runs from top left to bottom right, with a cadence of 16 min. The solar northwest limb is clearly visible and a flare arcade is evident in the latter half of the sequence. The corona above the flare is clearly depleted in the second half of the Fe XVI sequence. A white box defines the area analysed in the text. These are raw intensity images and are not differenced.

data does not show such a dramatic event but displays an initial rise and then a gradual fall off. This is actually reflected in the $\mathrm{He} \mathrm{I}$ and $\mathrm{O} \mathrm{V}$ data which suggests that we may be witnessing the eruption of a prominence in the brightening period (i.e. as
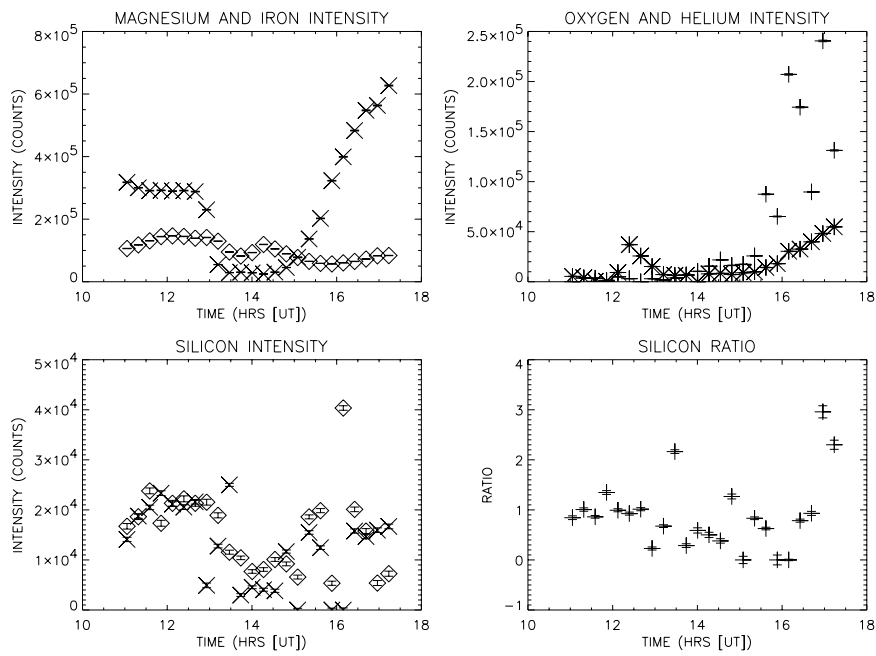

Fig. 6. The time-intensity profiles of the six emission lines for the boxed area of the July 25, 1999 event, displayed in a format similar to Fig. 2.

Table 4. The Event of July 25, 1999 (CDS Run 16947).

\begin{tabular}{|c|c|c|}
\hline Line & Dimming & Onset/Minimum \\
\hline He I $584 \AA$ & $-0 \%$ & $-1-$ \\
\hline No clear event. & & \\
\hline O V $629 \AA$ & $-0 \%$ & $-/-$ \\
\hline No clear event. & Brightens at 12:28 UT. & \\
\hline Mg IX $368 \AA$ & $-42 \%$ & $12: 55 / 13: 44 \mathrm{UT}$ \\
\hline Si X $347 \AA$ & $-64 \%$ & 12:55/14:00 UT \\
\hline Si X $356 \AA$ & $-86 \%$ & $13: 28 / 13: 44 \mathrm{UT}$ \\
\hline Fe XVI $360 \AA$ & $-89 \%$ & $12: 39 / 13: 28 \mathrm{UT}$ \\
\hline
\end{tabular}

DENSITY: Average fall in Si X ratio from about 1.3 to 0.5 through period from 11:00 UT to 14:00 UT, with one anomalous point at 13:28 UT. This represents a $94 \%$ density fall from $\log _{10}=9.4$ to $8.2\left(\mathrm{~cm}^{-3}\right)$.

MASS LOSS: DEM method $=7.4 \times 10^{11} \mathrm{~kg}, \mathrm{Si} X$ ratio method $=$ $3.4 \times 10^{12} \mathrm{~kg}$.

\section{POSITION ANGLE: 300-310 .}

LASCO EVENT: Large loop-like CME. Projected onset 13:20 UT. Position angle $232-360^{\circ}$. Mass $3.5 \times 10^{12} \mathrm{~kg}$.

COMMENT: Good CDS and LASCO coverage. Events appear to be well related.

it passes through our pixel box). Some corresponding dimming can be seen in the Si X data, but it is weaker. However, the Si X ratio does change considerably. As with the previous events, the nature of this event is summarised in Table 5.

We note that the initial density appears to be very high, though we are viewing a region which contains erupting (chromspheric) jet-like events, and loop systems with transition region and chromospheric temperatures and densities.

The LASCO CME and CDS dimming event position angles are consistent, especially when the non-radial nature of the jetlike events is considered. However, the EUV dimming mass is almost two orders of magnitude greater than the CME mass 
calculated from the LASCO observation. This is possibly due to the loss of cooler material in associated spray and prominence eruptive activity, seen as $\mathrm{He} \mathrm{I}$ and $\mathrm{O} \mathrm{V}$ jet-like events, some of which may have fallen back to the surface.

\subsection{August 19, 2000}

The August 19, 2000, events show the dimming of an off-limb loop system above the south-western limb in association with a narrow CME. The observations are shown, for the Fe XVI $360 \AA$ emission line in Fig. 9, in the same format as Fig. 1. In this event, the three CDS $4 \times 4$ arcmin fields are not aligned north-south but are offset to follow the south western limb, which can be seen in the upper frames.

The raw images of Fig. 9 show an active region system on the south western limb. The region consists of bright loop systems which can be seen on the limb, which runs from bottom left to top right of each mosaic frame, with the body of the Sun to the left. The lower panels show that the overlying, off-limb loops in a particular region darken considerably as the sequence progresses.

Again, we show a boxed region which is used to study the emission line time-intensity profiles, which are shown in Fig. 10. The box covers an area $60 \times 86$ pixels of size 2 arcsec $\times 1.68$ arcsec. The profiles show a dimming event of duration about 4-5 hours in the Mg IX data, in particular, after which the emission returns towards pre-event values. However, although the Fe XVI emission drops at the same time, it drops further in the later stages of the observation period. The He I and $\mathrm{O} \mathrm{V}$ data also show significant intensity declines and the shape of the Mg IX dimming is mimicked by the Si X $356 \AA$ profile. The characteristics are summarised in the table.

The dimming event and CME occupy similar but not identical position angles. The centrelines of the two are only $14^{\circ}$ apart and errors in positional measurement, especially of diffuse CME structures, as well as any non-radial motion, suggest that the two may well be related. The projected onset time of the CME, at 07:56 UT, is consistent with the dimming which is detected in the range 07:07 to 08:56 UT, depending on the emission line being analysed. The mass estimates of the CME and dimming are consistent. Thus, we believe that the two are indeed related, and the dimming is, again, showing the massloss from the CME source region.

\section{Analysis}

\subsection{Mass loss}

For each event, the mass lost from the low corona, which is indicated by the EUV dimming has been tabulated in Table 7 . Here, we compare the calculated EUV masses with the mass of the overlying CME events as seen in the LASCO data.

For the five events studied, the masses calculated from the dimming fell in the range $4.3 \times 10^{10}$ to $1.1 \times 10^{14} \mathrm{~kg}$ for the DEM method, and $1.3 \times 10^{11}$ to $2.7 \times 10^{14} \mathrm{~kg}$ for the Si X ratio method, with averages at $2.3 \times 10^{13} \mathrm{~kg}$ and $5.6 \times 10^{13} \mathrm{~kg}$, respectively. The Si X ratio method produces a higher value on each occasion, and this is, perhaps, surprising because the
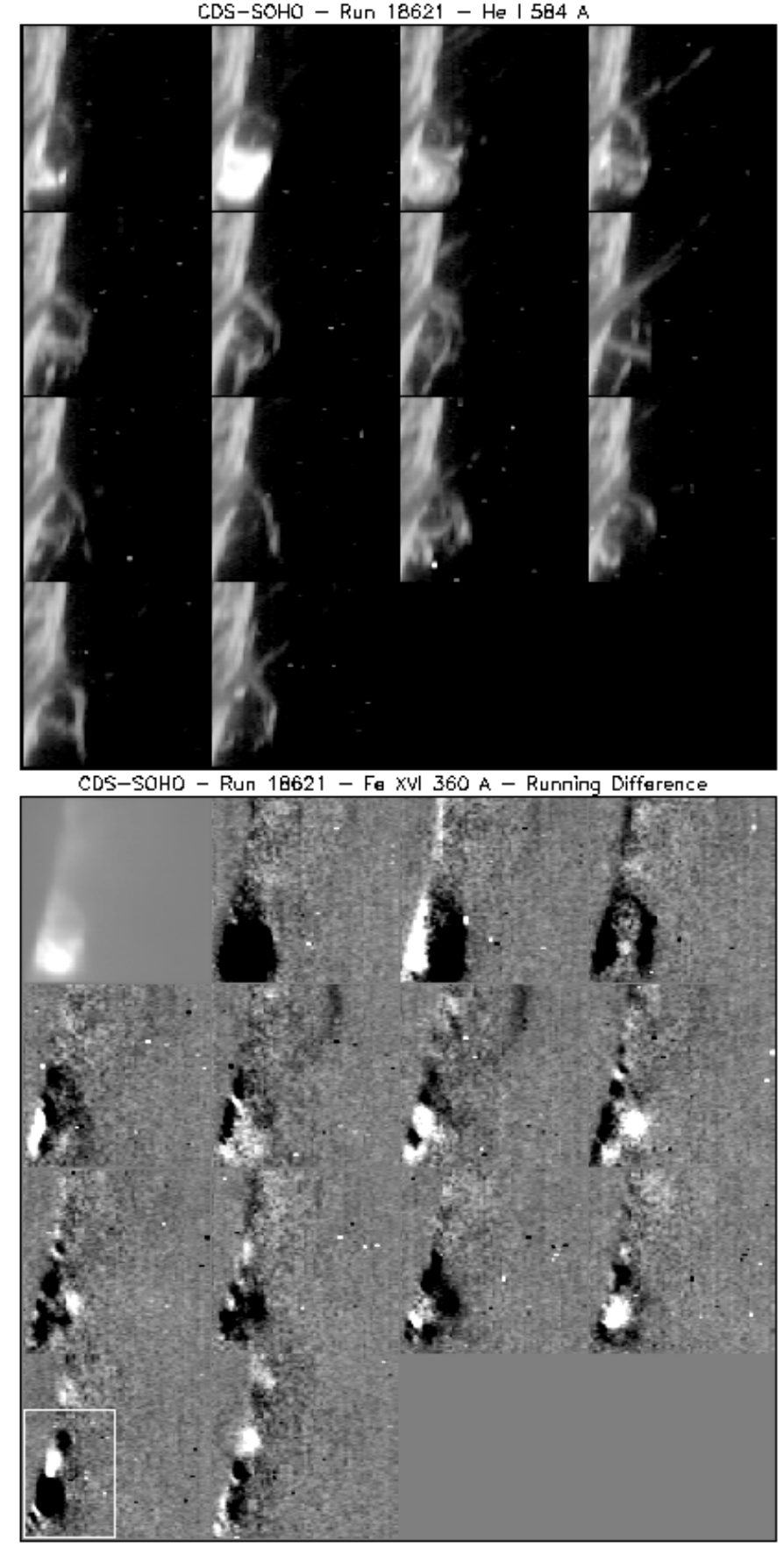

Fig. 7. The dimming event of February 19, 2000 detected in the $20000 \mathrm{He}$ I and 2 million K Fe XVI emission lines at $584 \AA$ and $360 \AA$, respectively. Only a $4 \times 4$ arcmin field is shown. The images run in sequence from top left to bottom right. The He I data (top panel) are raw images, which clearly show jet-like activity, a significant brightening in the second frame, and a lot of apparent motion. The Fe XVI images are differenced. The first Fe XVI image is shown in raw intensity and the rest are shown as a running difference.

DEM method takes into account the mass at temperatures out of the range for which the $\mathrm{Si} \mathrm{X}$ is sensitive. The Si X ratio mass is greater than the DEM mass by factors of 3.0, 3.8, 4.6, 2.5, and 2.8. They are all within a factor of 5 , which suggests that the mass estimates are reasonably consistent; the two methods are effectively confirming the results of one another. 

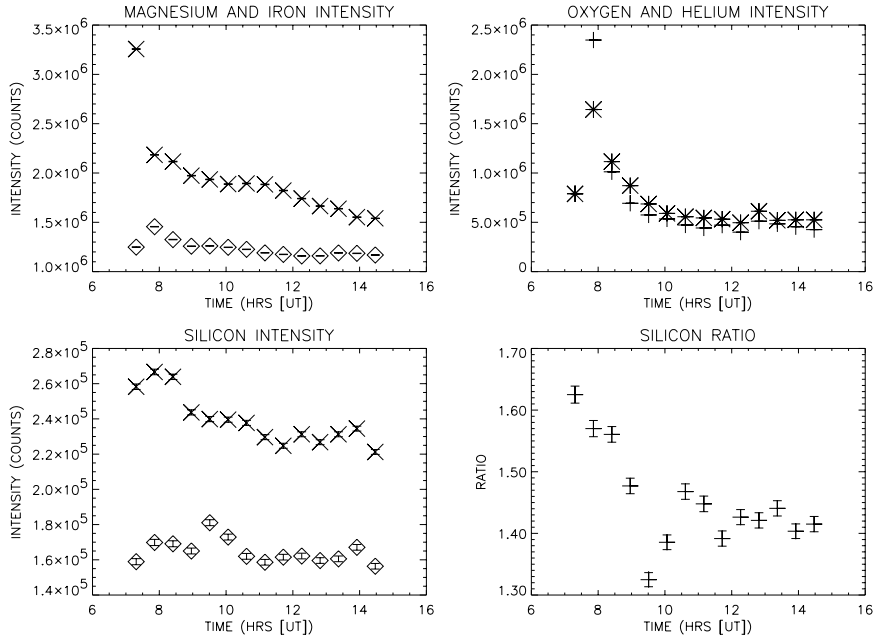

Fig. 8. The time-intensity profiles of the six emission lines for the dimming area of the February 19, 2000 event, displayed in a format similar to Fig. 2.

Table 5. The Event of February 19, 2000 (CDS Run 18621).

\begin{tabular}{lcl}
\hline \hline Line & Dimming & Onset/Minimum \\
\hline He I 584 & $-83 \%$ & $07: 51 / 11: 09$ UT \\
O V 629 & $-71 \%$ & $07: 51 / 12: 16$ UT \\
Mg IX 368 & $-20 \%$ & $07: 51 / 12: 16$ UT \\
Si X 347 & $-13 \%$ & $09: 30 / 11: 10$ UT \\
Si X 356 & $-16 \%$ & $07: 51 / 11: 43$ UT \\
Fe XVI 360 A & $-53 \%$ & $\leq 07: 18 / \geq 14: 28$ UT \\
Dramatic dimming at start & & \\
\hline
\end{tabular}

DENSITY: Steady fall, with some anomalous points, from Si X ratio of about 1.78 to 1.52 from 07:18 to 14:00. This represents a huge $95 \%$ density fall from $\log _{10}=11$ to $9.7\left(\mathrm{~cm}^{-3}\right)$, with the early values suggesting extreme densities.

MASS LOSS: DEM method $=1.1 \times 10^{14} \mathrm{~kg}$, Si X ratio method $=$ $2.7 \times 10^{14} \mathrm{~kg}$.

\section{POSITION ANGLE: 250-260 .}

LASCO EVENT: Loop ejection with trailing structure. First observed at 08:30 UT. Projected onset 07:40 UT. Position angle 260-292 . Mass $1.1 \times 10^{12} \mathrm{~kg}$.

COMMENT: Good CDS and LASCO coverage. Events appear to be well related.

CME masses are usually quoted as being in the range $2 \times 10^{11}$ to $2 \times 10^{13} \mathrm{~kg}$ (see e.g. Hundhausen 1997; Howard et al. 1985). However, the lower limit, in particular, is certainly an instrumental limit and one might realistically expect that there is no lower limit to a CME mass. In addition, it is almost certain that larger CME masses can be found. However, it is interesting to note that the dimming masses are consistent with the CME mass range quoted above, but extend slightly beyond it on either extreme. The average DEM and Si X ratio dimming mass is consistent with the mass of a more massive CME.

The ratios of the estimated CME and DEM dimming masses for the five events studied are 1.2, 0.3, 4.7, 0.01, 0.7. From the Si X mass estimates, the ratios are 0.4, 0.07, 1.0,
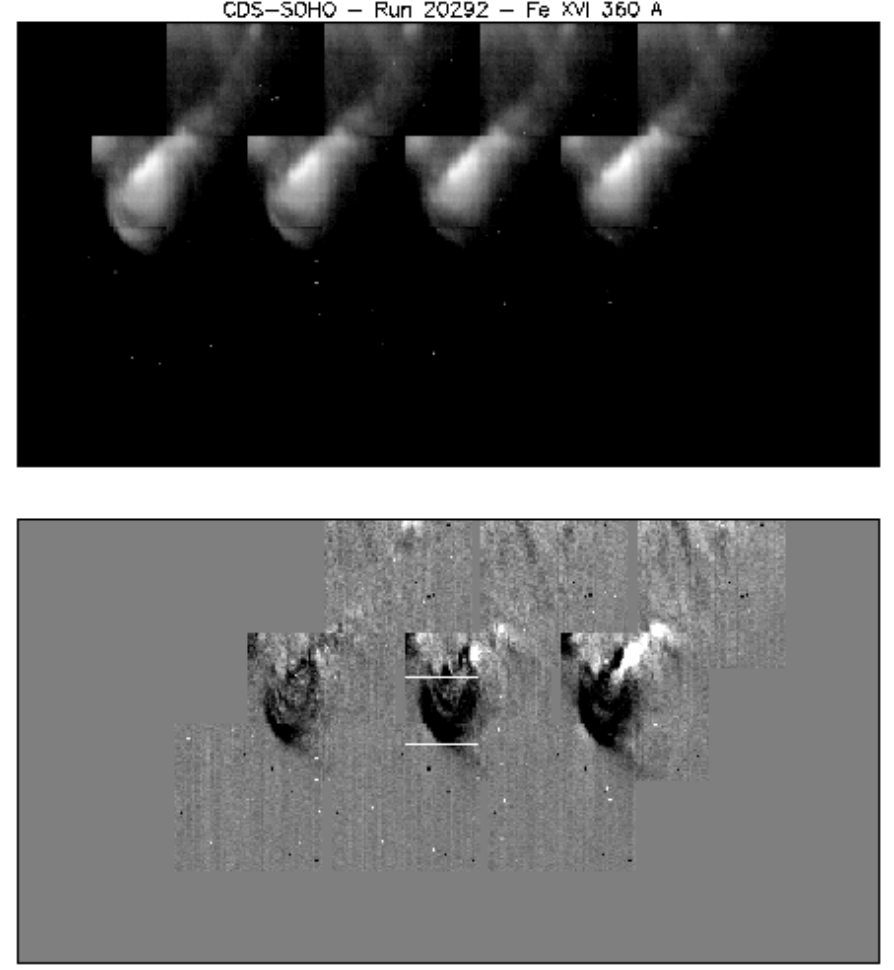

Fig. 9. The dimming event of August 19, 2000 detected in the 2 million K Fe XVI emission line at $360 \AA$, displayed in the same format as Fig. 1.
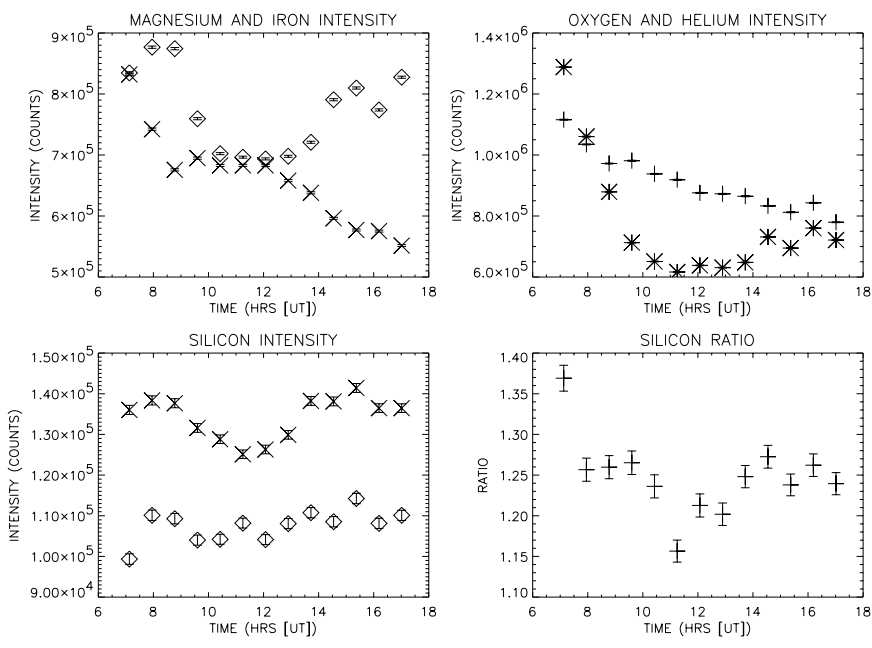

Fig. 10. The time-intensity profiles of the six emission lines for the dimming area of the August 19, 2000 event, displayed in a format similar to Fig. 2.

$0.004,0.3$. All are within a factor of 10 (range 0.1 to 10 ), except the February 19 event and the Si X calculation for the May 8/9 event. It is noticeable that the events for which the CME mass is much less than the estimated dimming mass (May 8/9, February 19 and August 19) are the three events where ejected (dimming of) He I/O V material was detected. This suggests that the white-light LASCO estimates do not account for the full mass-loss of the cooler material. Since a coronagraph is sensitive to density and not temperature, one conclusion would be that some mass falls back to the surface. 
Table 6. The Event of August 19, 2000 (CDS Run 20292).

\begin{tabular}{|c|c|c|}
\hline Line & Dimming & Onset/Minimum \\
\hline $\begin{array}{l}\text { He I } 584 \AA \\
\text { Continuous decline. }\end{array}$ & $-30 \%$ & $\leq 07: 07 / \geq 17: 00 \mathrm{UT}$ \\
\hline O V $629 \AA$ & $-52 \%$ & $\leq 07: 07 / 11: 15 \mathrm{UT}$ \\
\hline $\begin{array}{l}\text { Mg IX } 368 \AA \\
\text { Brightens after 13:00 UT }\end{array}$ & $-21 \%$ & 08:46/12:04 UT \\
\hline Si X $347 \AA$ & $-5 \%$ & 07:57/09:36 UT \\
\hline $\begin{array}{l}\text { Si X } 356 \AA \\
\text { Similar to Mg IX }\end{array}$ & $-10 \%$ & 07:57/11:15 UT \\
\hline $\begin{array}{l}\text { Fe XVI } 360 \AA \\
\text { Stays low then falls }\end{array}$ & $-19 \%$ & $\leq 07: 07 / 08: 46 \mathrm{UT}$ \\
\hline
\end{tabular}

DENSITY: Average value of $\mathrm{Si} \mathrm{X}$ ratio at 1.42 falls to 1.29 at 11:15 UT. However, first data point gives 1.56. Assuming first point is anomalously high, density falls $37 \%$ from $\log _{10}=9.5$ to $9.3\left(\mathrm{~cm}^{-3}\right)$.

MASS LOSS: DEM method $=6.4 \times 10^{11} \mathrm{~kg}$, Si $X$ ratio method $=$ $1.8 \times 10^{12} \mathrm{~kg}$.

POSITION ANGLE: 221-231 .

LASCO EVENT: Narrow "Faint jet" ejection. First observed at 08:30 UT. Projected onset 07:56 UT. Position angle 237-245 . Mass $4.7 \times 10^{11} \mathrm{~kg}$.

COMMENT: Good CDS and LASCO coverage. Events seem to be well related.

Having said that, the CME masses are calculated assuming that the event is in the plane of the sky and this, combined with any mass loss due to cooler, unseen material, means that the error on such a calculation may be considered to be occasionally up to an order of magnitude. The error on the EUV mass calculations must be considered to be of the same order; the temperature coverage is restricted and the calculations are made using a standard DEM and making assumptions about geometry out of the plane of the sky. Thus, the fact that most of the estimates are within an order of magnitude is highly suggestive that the EUV dimming is a signal of mass loss from a source region which is intimately associated with the source of the CME mass. One conclusion would be that the dimming identifies the mass which is to become the CME, or a substantial fraction of the mass, which subsequently forms the mass detected as the CME. An alternative explanation is that the material we identify in the dimming goes to replace the mass ejected in the $\mathrm{CME}$, i.e. it replenishes the material in the medium altitude corona.

\subsection{Timing}

We now consider the relative timing of the dimming events and the projected onsets of the associated CMEs. The timing information is given in the tables above.

The projected CME onsets assume a zero altitude onset at the limb. A zero altitude onset is unrealistic; the CME source region must have some altitude. Thus, we would expect the CME onset to be later than the projected onset. However, the source region may not be on the limb and there must be some
Table 7. Summary of the EUV dimming masses and the CME mass.

\begin{tabular}{lll}
\hline \hline Date & $\begin{array}{l}\text { EUV dimming mass }(\mathrm{kg}) \\
\text { DEM/Si X methods }\end{array}$ & $\begin{array}{l}\text { LASCO CME } \\
\text { mass }(\mathrm{kg})\end{array}$ \\
\hline Jul. 16 1997 & $4.3 \times 10^{10} / 1.3 \times 10^{11}$ & $5 \times 10^{10}$ \\
May 8/9 1999 & $1.1 \times 10^{12} / 4.2 \times 10^{12}$ & $3 \times 10^{11}$ \\
Jul. 25 1999 & $7.4 \times 10^{11} / 3.4 \times 10^{12}$ & $3.5 \times 10^{12}$ \\
Feb. 19, 2000 & $1.1 \times 10^{14} / 2.7 \times 10^{14}$ & $1.1 \times 10^{12}$ \\
Aug. 19, 2000 & $6.4 \times 10^{11} / 1.8 \times 10^{12}$ & $4.7 \times 10^{11}$ \\
\hline
\end{tabular}

early acceleration of the ascending CME material. These would push the projected onset time earlier. Thus, the zero altitude projected onset is probably as good as we can produce, but it must be assumed that there is an error of several tens of minutes.

For all five events, the CME onset is projected to be within about 30 min of the dimming onset or is within the time-span during which different EUV wavelength bands displayed dimming. Thus, there is a superb association between the dimming events and CME onsets and one must conclude that they are coincident. Again, this strengthens the argument that the dimming region is the region from which the CME source material is extracted.

However, another aspect of the timing is the fact that the decay in intensity for the EUV dimming events extends over many hours which, in itself, seems incompatible with such an impulsive event as a CME. If one examines the Mg IX intensity curves for the five events, one finds times from the dimming onset to the minimum of intensity ranging from over 12 hours to $49 \mathrm{~min}$. Admittedly, the 12 hour decay (July 16) showed the most significant portion of the decay in the first few hours. However, any CME onset models which include aspects of the dimming, must cater for the long time-scale of the depletion.

If one imagines that the dimming mass is identical to the mass seen later as the $\mathrm{CME}$, then the dimming region ought to recover as the corona above recovers. However, for the July 16 event, the lower corona showed continued dimming for some hours after the coronagraph observation indicated a return to pre-event conditions. This is most likely a similar situation to that of February 19. However, the July 25 and August 19 dimmings show signs of recovery, or filling in, of the EUV dimming region after a period of about 2 hours. For this reason, one might conclude that there are two possibilities. First, we may be witnessing mass loss as part of the ascending CME and a subsequent recovery. Second, the CME may erupt, involving nearby, large portions of the corona and what we witness is the redistribution of mass underneath after the CME onset, which can last for many hours. In the latter case, we woud be witnessing the aftermath of the CME in the underlying corona. We may be witnessing some degree of both scenarios for many events. However, in either case, the dimming characteristics provide information on the CME process. 


\subsection{Temperature}

The dimming characteristics of each of the temperatures represented by the different emission lines can vary considerably. A summary of the characteristic dimming of each line (temperature) is given in Table 8.

In 2 out of the 5 events considered, there was no clear dimming in the $\mathrm{He}$ and $\mathrm{O} \mathrm{V}$ intensities, and in one further case, no $\mathrm{O} \mathrm{V}$ dimming. However, for the remaining events the He I and $\mathrm{O} \mathrm{V}$ dimming was significant enough to result in an average dimming of $30 \%$ and $25 \%$, respectively. The fact that these cooler lines (He I and O V) appear as either zero dimming or high dimming values may relate to associated prominence or spray eruptions.

Although for the Mg IX and both Si X lines there were no cases with no clear dimming, for all six emission lines the average dimming was in the range $23-42 \%$. The Fe XVI emission line displays the highest average dimming, at $42 \%$.

In general, we can state that the coronal temperatures commonly show a dimming of order $20-40 \%$ with values as high as $89 \%$. However, it is clear from the data presented in the tables above that the degree of dimming in any one temperature, e.g. Mg IX, is not necessarily an indicator of the degree of dimming expected in another line, e.g. Fe XVI. This means that the temperature of the ejected material can be selected in some way. This was pointed out by Harrison \& Lyons (2000).

\subsection{Location}

Four out of five of the events have dimming position angles which underlie the CME position angle span. For the remaining event, the dimming site lies $6^{\circ}$ away from the CME span. The centre-lines of the dimming event position angle locations and the spans of the associated CMEs are separated by $3^{\circ}, 4.5^{\circ}, 9^{\circ}$, $21^{\circ}$ and $15^{\circ}$. When taken in conjunction with the timings and mass calculations, there is clear evidence that the events are intimately related. Given the fact that the boundaries of many CME-spans and the dimming regions are not sharply defined, and that there may be non-radial motion, we consider all five dimming events to be well associated with the overlying CMEs.

\section{Discussion and conclusions}

This is the first study of the plasma diagnostic characteristics of a set of EUV dimming events associated with CME onsets. From the analysis if the five events of this paper, a set of useful conclusions can be made:

1. The EUV dimming events are shown to the due to massloss, and calculations of the mass extracted from the dimming regions for the events under study are typical of CME masses. This result alone is highly suggestive that the dimming is a signal of the source region of the CME mass.

2. The comparison of mass-loss calculated for individual events to the mass of associated CMEs appears to confirm that the dimming is indeed due to the extraction of the mass. This mass may form a substantial fraction of the mass which is ejected in the CME, or it may be mass which is lost due to
Table 8. Summary of the intensity of EUV dimming, given as percentages of the initial intensity.

\begin{tabular}{lll}
\hline \hline Ion & $\begin{array}{l}\text { Average dimming } \\
\text { (\% of initial intensity) }\end{array}$ & $\begin{array}{l}\text { Dimming range } \\
\text { (\% of initial intensity) }\end{array}$ \\
\hline He I & 30 & 0 to 83 (2 cases of 0$)$ \\
O V & 25 & 0 to 71 (3 cases of 0$)$ \\
Mg IX & 28 & 14 to 42 (no cases of 0$)$ \\
Si X 347 & 23 & 5 to 64 (no cases of 0$)$ \\
Si X 356 & 30 & 10 to 86 (no cases of 0$)$ \\
Fe XVI & 42 & 0 to $89(1$ case of 0$)$ \\
\hline
\end{tabular}

replenishment of mass lost in the CME from another part of the corona.

3. Continued dimming over many hours for some events suggests that the low coronal dimming, at least for some events, may be due to the restructuring of the corona in the aftermath of the CME.

4. It is important to note that the comparison of emission lines show that the dimming is due to a mass change and not a heating or cooling event in the coronal plasma.

5. The EUV dimming onset and CME onset are intimately associated, and, given the uncertainties in the onset times, the case is made that the dimming onset and CME onset are indeed coincident.

6. The decay in intensity of a dimming event extends over many hours, despite the much shorter duration of the overlying CMEs.

7. Typically, emission lines show dimming of order 23$42 \%$ on average during a dimming event, and this is shown for emission lines in the temperature range 20000 to $2000000 \mathrm{~K}$. The greatest average drop in intensity was detected for the hottest line (Fe XVI at $2000000 \mathrm{~K}$ ).

8 . The degree of dimming can vary considerably and values from $0 \%$ to $90 \%$ are detected, with the intensity decrease in different lines (temperatures) varying considerably for the same event, i.e. there is some temperature "selection".

9. In general, the locations of the dimming events are directly under the CME or to one side of the CME-span.

First and foremost, these results indicate that we can identify the source region of a CME using the detection of EUV dimming. This in itself is important for several reasons. It provides a method for identification of the magnetic topology of the source region. This cannot be done using Zeeman splitting methods and wide-band imaging is not necessarily appropriate (see Sect. 1). This topology must be used for model development. Some dimming events, such as July 16, 1997, May 8/9, 1999, February 19, 2000 are rather diffuse and one would have to surmise that they are due to ascending diffuse coronal loops, but the dimming events of July 25, 1999, and August 19, 2000 clearly show loop like structures. The July 25 observation showed loops in the corona which disappeared, and the August 19 dimming was itself clearly seen in the shape of a loop structure. Further refinement of the dimming analysis, with careful line selection and better cadence, may be used in future for a good identification of the magnetic structure of the CME source. 
Identifying mass loss through EUV dimming is also important because it can be used to identify events on the disc, i.e. CME events which are potentially Earth-directed. Without coronagraphs out of the Sun-Earth line, and, given the diffuse nature of Earth-directed "halo" CMEs, this method may be extremely valuable for CME prediction.

With regard to extending our understanding of the CME onset process, we must now take several of the results above into account, other than the potential for the identification of the basic magnetic structure. We do not witness an impulsive dimming process, but a gradual one, with the extraction of significant mass from low corona features over many hours. Does this suggest a gradual CME driver, e.g. continued shear or slow flux injection, rather than an impulsive trigger such as magnetic reconnection? Does this support, for example, the continuous shear model approach due to Steinolfsen (1991), or a long-duration flux injection approach due to Krall et al. (2001) rather than the more impulsive reconnection approach, such as in the "breakout" model due to Antiochos et al. (1999)? On the other hand, is it possible that there is a more impulsive CME onset but the low-coronal response is slow and extends over a long period after the event?

The latter is probably true because models requiring a longduration, gradual drivers would most likely result in gradual $\mathrm{CME}$ and dimming signatures and our concern here is the apparent mismatch between the CME duration and the longerduration dimming event.

However, we may be seeing both. The July 25, 1999 event saw rapid dimming with clear pre-event coronal loops. The July 16, 1997 event showed a long-duration dimming. From the observational side, we must follow up these observations with attempts at higher cadence spectroscopic observations and that may have to wait for future missions. If there is a trigger, such as reconnection, it might be detected in the EUV, through the presence of small bidirectional jets and associated hot-spots. The basic cadence of our observations may be slow, but for some events in the observation campaigns, we may witness such signatures. Work is proceeding on this at this time.

CME models must explain also the wide variation in characteristics at differing temperatures. In some events we see dimming at the hottest temperatures and in others the events appear to be dominant at about 1 million $\mathrm{K}$. The signatures in the $\mathrm{He} \mathrm{I}$ and $\mathrm{O} \mathrm{V}$ data appear to relate to prominence or cool EUV spray events.

In effect, these first attempts at deriving the plasma characteristics of dimming events, in relation to CME activity, have provided some basic results about mass-loss and CME source location, but there are many open questions which require attention and, for this reason, the campaign continues.

Acknowledgements. CDS was built and is operated by a consortium led by the Rutherford Appleton Laboratory and including the Mullard Space Science Laboratory, the NASA Goddard Space Flight Center, Oslo University and the Max Planck Institute for Extraterrestrial Physics, Garching. LASCO was built and is operated by a consortium led by the US Naval Research Laboratory and including Birmingham University, the Max Planck Institute for Aeronomy, Lindau, and the Laboratoire d'Astronomie Spatiale, Marseille. SOHO is a mission of international cooperation between ESA and NASA.

\section{References}

Antiochos, S. K., DeVore, C. R., \& Klimchuk, J. A. 1999, ApJ, 510, 485

Biesecker, D. A., Myers, D. C., Thompson, B. J., Hammer, D. M., \& Vourlidas, A. 2002, ApJ, 569, 1009

Brueckner, G. E., Howard, R. A., Komen, M. J., et al. 1995, Sol. Phys., 162,357

Harrison, R. A. 1997, in Proc. 31st ESLAB Symp., ESA SP-415, 121

Harrison, R. A., Sawyer, E. C., Carter, M. K., et al. 1995, Sol. Phys., 162,233

Harrison, R. A., \& Lyons, M. 2000, A\&A, 358, 1097

Harrison, R. A., Bryans, P., \& Bingham, R. 2001, A\&A, 379, 324

Howard, R. A., Sheeley, N. R., Koomen, M. J., \& Michels, D. J. 1985, J. Geophys. Res., 90, 8173

Howard, R. A., Brueckner, G. E., St Cyr, C., et al. 1997, in Coronal Mass Ejections, ed. N. Crooker, J. Joselyn, \& J. Feynman, Geophys. Monograph Ser., AGU, 17

Hundhausen, A. J. 1997, in Coronal Mass Ejections, ed. N. Crooker, J. Joselyn, \& J. Feynman, Geophys. Monograph Ser., AGU, 1

Krall, J., Chen, J., Duffin, R. T., Howard, R. A., \& Thompson, B. J. 2001, ApJ, 562, 1045

Low, B. C. 1981, ApJ, 251, 352

Mason, H. E., Young, P. R., Pike, C. D., et al. 1997, Sol. Phys., 170, 143

Priest, E. R. 1988, ApJ, 328, 848

Raymond, J., \& Doyle, J. G. 1981, ApJ, 247, 686

Steinolfson, R. S. 1991, ApJ, 382, 677

Sterling, A. C., \& Hudson, H. S. 1997, ApJ, 491, L55

Thompson, B. J., Gurman, J. B., Neupert, W. M., et al. 1999, ApJ, 517, L151

Wolfson, R. L. T. 1982, ApJ, 255, 774

Zarro, D. M., Sterling, A. C., Thompson, B. J., Hudson, H. S., \& Nitta, N. 1999, ApJ, 520, L139 